J Sci.Univ.Kelaniya 4 (2008): 1-10

\title{
APPLIED MAGNETIC FIELD AND STRESS INDUCED ANISOTROPY DEPENDENCE OF ENERGY FOR ORIENTED FERROMAGNETIC THICK FILMS
}

P. SAMARASEKARA

\section{Department of Physics, University of Ruhuna, Matara, Sri Lanka}

E-mail: pubudus@phy.ruh.ac.lk

\section{ABSTRACT}

The energy of perfectly oriented thick ferromagnetic films at different applied magnetic field and stress was studied using classical model of Heisenberg Hamiltonian. For the values of parameters used in this report, the energy required to orient the film with 1000 layers in $41.4^{\circ}$ or $57.6^{\circ}$ directions becomes minimum at applied magnetic fields of $\frac{\boldsymbol{H}_{\text {out }}}{\boldsymbol{\omega}}=4.8$ or $\frac{\boldsymbol{H}_{\text {in }}}{\boldsymbol{\omega}}=4.8$, respectively. The film can be easily oriented in $172^{\circ}$ direction by applying the external stress $\frac{\boldsymbol{K}_{\boldsymbol{s}}}{\boldsymbol{\omega}}=3.6$ perpendicular to the film plane, for the values of parameters used in this report. These final results mainly depend on the values of other parameters used for calculations. The energy becomes minimum and maximum at almost the same values of oriented angle $(\theta)$, in plane magnetic field, out of plane magnetic field and stress for both $\mathrm{sc}(001)$ and bcc(001 lattice types. But the values of energies corresponding to maxima and minima are slightly different for the sc(001) and bcc(001) lattices.

Keywords: Magnetic thin films, ferromagnetism, Heisenberg Hamiltonian, stress induced anisotropy and spin

\section{INTRODUCTION}

The solution of Hamiltonian for ferromagnetic thin films has been obtained using 2D XY model (Zhao et al., 2002), two-spin mean field theory (Jensen \& Dreysse, 2002), spin half Ising model (Bentaleb et al., 2002) and classical Heisenberg 


\section{P Samarasekara}

model (Shan-Ho et al., 2003; Usadel \& Hucht, 2002) by some other researchers. In addition to this, the Hamiltonian in Heisenberg model has been solved using Green functions (Ze-Nong et al., 1993). Although most of the films available in the applications of magnetic memory and microwave devices are thick films, no sufficient researches have been done on solution Heisenberg Hamiltonian of oriented ferromagnetic thick films.

The classical Heisenberg Hamiltonian has been used to derive an equation for the energy of oriented ferromagnetic thin films in a previous publication (Samarasekara, 2006). The energy has been calculated for discrete and continuous cases using a summation and an integral, respectively. All the results obtained using summation and integral were exactly the same. The variation of energy with number of layers and azimuthual angle of spin was studied for $\mathrm{sc}(001)$ and bcc(001) lattice structures earlier. Also the energy curves for thick films with $\mathrm{N}=10000$ has been given for these two lattice structures (Samarasekara, 2006). The angle between easy and hard directions was also found to be $90^{\circ}$ according to previous publications (Samarasekara, 2006). The stress induced anisotropy plays a vital role in the investigations of soft ferromagnetic materials since the stress induced anisotropy is considerable compared with the crystal anisotropy (Samarasekara \& Cadieu, 2001). Therefore the stress induced anisotropy has been taken into account for the studies given here. Under the influence of an applied magnetic field, the demagnetization effect takes place inside a magnetic thin film due to the separation of magnetic poles. But the dipole interaction takes place even without the applied field. Therefore, the origins of the dipole interaction and the demagnetization factor are different. Although the dipole interaction is a microscopic parameter, demagnetization factor is a macroscopic parameter. Therefore, both dipole interaction and demagnetization effect have been considered for these studies.

\section{MODEL AND DISCUSSION}

The Hamiltonian in Heisenberg model can be written in the following form for a ferromagnetic thin film. 


$$
\begin{aligned}
\mathrm{H} & =-\frac{J}{2} \sum_{m, n} \vec{S}_{m} \vec{S}_{n}+\frac{\omega}{2} \sum_{m \neq n}\left(\frac{\vec{S}_{m} \vec{S}_{n}}{r_{m n}{ }^{3}}-\frac{3\left(\vec{S}_{m} \cdot \vec{r}_{m n}\right)\left(\vec{r}_{m n} \cdot \vec{S}_{n}\right)}{r_{m n}{ }^{5}}\right)-\sum_{m} D_{\lambda_{m}}{ }^{(2)}\left(S_{m}{ }^{2}\right)^{2}-\sum_{m} D_{\lambda_{m}}{ }^{(4)}\left(S_{m}{ }^{z}\right)^{4} \\
& -\sum_{m, n}\left[\vec{H}-\left(N_{d} \vec{S}_{n} / \mu_{0}\right)\right] \cdot \vec{S}_{m}-\sum_{m} K_{s} \operatorname{Sin} 2 \theta_{m}
\end{aligned}
$$

Here, first, second, third and fourth terms represent the spin exchange interaction, magnetic dipole interaction, second order anisotropy and fourth order anisotropy, respectively. The indices of planes are denoted by $\mathrm{m}$ and $\mathrm{n}$, and they vary from 1 to $\mathrm{N}$ for a film with $\mathrm{N}$ number of layers. $\vec{S}_{i}$ is a spin vector with unit length at point $\vec{r}_{i}$ in layer $\lambda_{i}$. Therefore the ground state energy will be calculated per spin with Z-axis normal to film plane. $\vec{H}$ and $N_{d}$ are the external magnetic field and the demagnetization factor with the effective magnetic moment $\mu$ of the spins incorporated. $\omega=\frac{\mu_{0} \mu^{2}}{4 \pi a^{3}}$ is the strength of long range dipole interaction on a lattice with lattice constant a, where $\mu_{0}$ is the permeability. $r_{m n}$ is the separation between two spins $\mathrm{s}_{\mathrm{m}}$ and $\mathrm{s}_{\mathrm{n}} . D_{\lambda_{m}}{ }^{(2)}$ and $D_{\lambda_{m}}{ }^{(4)}$ are second and fourth order magnetic anisotropy constants of the lattice, respectively. Here, $\mathrm{N}_{\mathrm{d}}$ given in $5^{\text {th }}$ term represents the demagnetization factor, and the last term indicates the change of magnetic energy under the influence of a stress. $\mathrm{K}_{\mathrm{s}}$ depends on the product of magnetostriction coefficient $\left(\lambda_{s}\right)$ and the stress $(\sigma)$. Here $\theta$ is the angle between local magnetization (M) and the stress. Within a single domain, $\mathrm{M}$ is parallel to the spin. If stress is applied normal to the film plane, then $\theta_{\mathrm{m}}$ is the angle between a perpendicular line drawn to the film plane and the local spin. For a thin film, $\mathrm{N}_{\mathrm{d}}=1$ in perpendicular direction and $\mathrm{N}_{\mathrm{d}}=0$ in film plane in SI units. Here $\mathrm{K}_{\mathrm{s}}$ can be positive or negative depending on the type of stress whether it is compressive or tensile.

The energy of oriented ferromagnetic thick films has been derived in the following form using summation for discrete case (Samarasekara, 2006),

$$
\begin{aligned}
E(\theta) & =-\frac{J}{2}\left[N Z_{0}+2(N-1) Z_{1}\right] \\
& +\left\{N \Phi_{0}+2(N-1) \Phi_{1}+2 a e^{-2 b}\left[\frac{N-2+e^{-b}(1-N)}{\left(1-e^{-b}\right)^{2}}\right]\right\}\left(\frac{\omega}{8}+\frac{3 \omega}{8} \cos 2 \theta\right) \\
& -\cos ^{2} \theta \sum_{m=1}^{N} D_{m}{ }^{(2)}-\cos ^{4} \theta \sum_{m=1}^{N} D_{m}^{(4)}-N\left(H_{\text {in }} \sin \theta+H_{\text {out }} \cos \theta-\frac{N_{d}}{\mu_{0}}+K_{s} \sin 2 \theta\right)
\end{aligned}
$$


For $\operatorname{sc}(001)$ lattice with $\mathrm{Z}_{0}=4, \mathrm{Z}_{1}=1, \Phi_{0}=9.0336, \Phi_{1}=-0.3275, \frac{J}{\omega}=1.06 \times 10^{3}$, $\mathrm{a}=-16 \pi^{2} \quad \mathrm{~b}=2 \pi$ and $\mathrm{N}=1000$ (Usadel \& Hucht, 2002), $\mathrm{E}(\theta)=-3179 \times 10^{3} \omega+1047$ $\omega(1+3 \cos 2 \theta)$

$$
\begin{aligned}
& -\cos ^{2} \theta \sum_{m=1}^{N} D_{m}{ }^{(2)}-\cos ^{4} \theta \sum_{m=1}^{N} D_{m}^{(4)}-1000\left(H_{\text {in }} \sin \theta+H_{\text {out }} \cos \theta-\frac{N_{d}}{\mu_{0}}+K_{s} \sin 2 \theta\right) \\
& \text { When } \frac{\sum_{d}^{N} D_{m}{ }^{(2)}}{\mu_{0} \omega}=10, \frac{\sum_{m=1}^{N} D_{m}{ }^{(4)}}{\omega}=4000, \frac{m_{m=1}}{\omega}=189, \mathrm{H}_{\mathrm{in}}=0 \text { and } \frac{K_{s}}{\omega}=5 \\
& \frac{E(\theta)}{\omega}=-3168 \times 10^{3}+3141 \cos 2 \theta-4000 \cos ^{2} \theta-189 \cos ^{4} \theta-000\left(\frac{\boldsymbol{H}_{\text {out }}}{\omega} \cos \theta+5 \sin 2 \theta\right)
\end{aligned}
$$

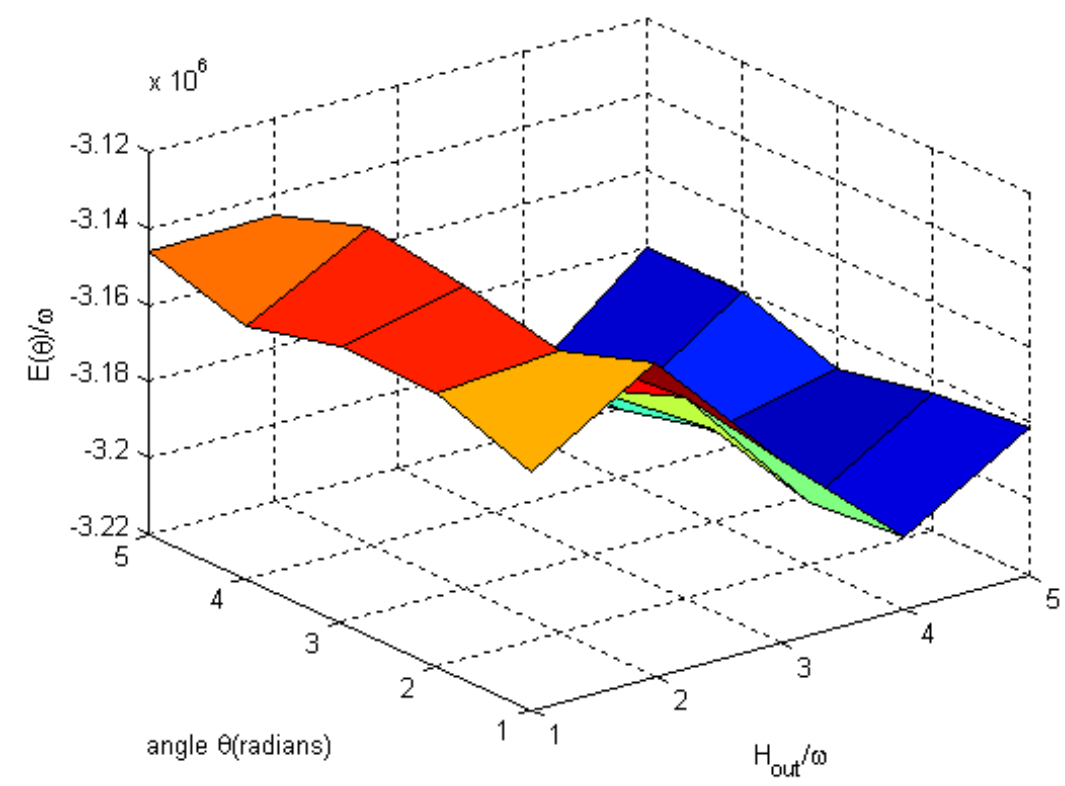

Figure 1: $\quad 3$-D graph of $\frac{E(\theta)}{\omega}$ versus $\frac{H_{o u t}}{\omega}$ and angle for $\operatorname{sc}(001)$ film with 1000 layers.

All the parameters $\mathrm{J}, \omega, \mathrm{N}_{\mathrm{d}}, \mathrm{D}_{\mathrm{m}}{ }^{(2)}, \mathrm{D}_{\mathrm{m}}{ }^{(4)}, \mathrm{H}_{\mathrm{in}}, \mathrm{H}_{\text {out }}$ and $\mathrm{K}_{\mathrm{s}}$ are independent of each other for any particular magnetic material. The 3-D graph of $\frac{\boldsymbol{E}(\boldsymbol{\theta})}{\omega}$ versus $\frac{\boldsymbol{H}_{\text {out }}}{\boldsymbol{\omega}}$ and angle is given in figure 1 . The energy required to orient the film in some certain directions becomes minimum at $\frac{\boldsymbol{H}_{\text {out }}}{\boldsymbol{\omega}}=4.8$. This implies that the thin film with 1000 
layers can be easily oriented in that certain direction by applying this external magnetic field in perpendicular directions.

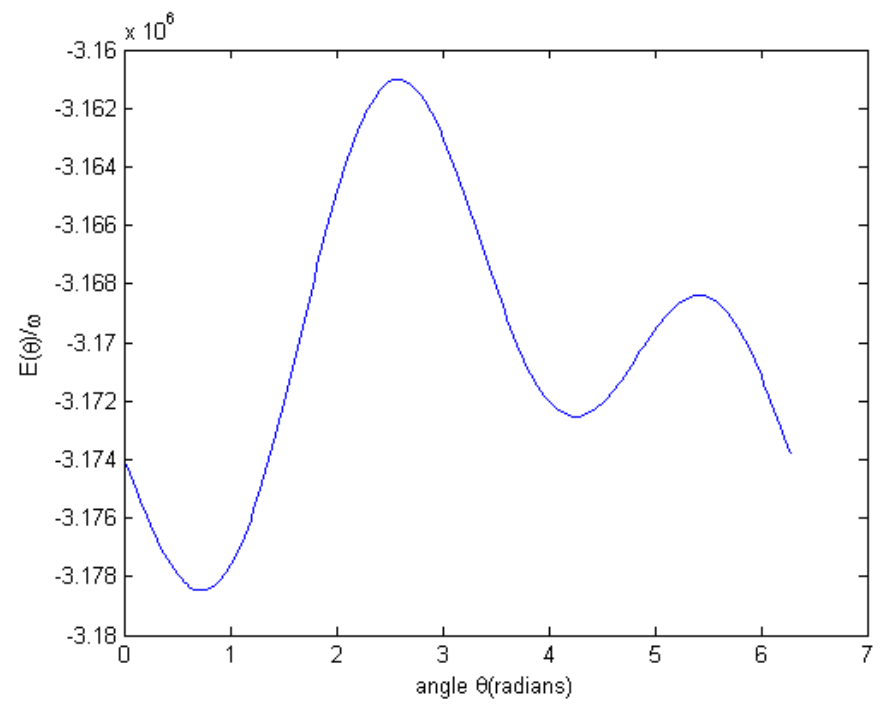

Figure 2: Graph between energy and the angle for sc(001) film with $\mathrm{N}=1000$.

At $\frac{\boldsymbol{H}_{\text {out }}}{\boldsymbol{\omega}}=4.8$, the graph between energy and the angle is given in figure 2 in order to find the angle corresponding to minimum energy. The energy is minimum and maximum at $41.4^{\circ}$ and $147.7^{\circ}$, respectively. Therefore, the film with 1000 layers can be easily oriented along direction of $41.4^{\circ}$ by applying this magnetic field.

The equation for energy derived using integral for continuous case can be given as (Samarasekara, 2006),

$$
\begin{aligned}
& E(\theta)=-\frac{J}{2}\left[N Z_{0}+2(N-1) Z_{1}\right] \quad+\left[N \Phi_{0}+2(N-1) \Phi_{1}\right. \\
& +\frac{2}{N-3}\left\{\frac{N a}{b}\left[e^{-2 b}-e^{-b(N-1)}\right]+\frac{a}{b}\left[(N-1) e^{-b(N-1)}-2 e^{-2 b}\right]\right. \\
& \left.\left.-\frac{a}{b^{2}}\left[e^{-2 b}-e^{-b(N-1)}\right]\right\}\right]\left(\frac{\omega}{8}+\frac{3 \omega}{8} \cos 2 \theta\right) \\
& -\cos ^{2} \theta \sum_{m=1}^{N} D_{m}^{(2)}-\cos ^{4} \theta \sum_{m=1}^{N} D_{m}^{(4)}-N\left(H_{\text {in }} \sin \theta+H_{\text {out }} \cos \theta-\frac{N_{d}}{\mu_{0}}+K_{s} \sin 2 \theta\right)
\end{aligned}
$$

The energy found using this equation 4 is exactly the same as the energy given in above equation 3 for discrete case. Therefore, all the derivation given in this contribution was performed only for discrete case. 


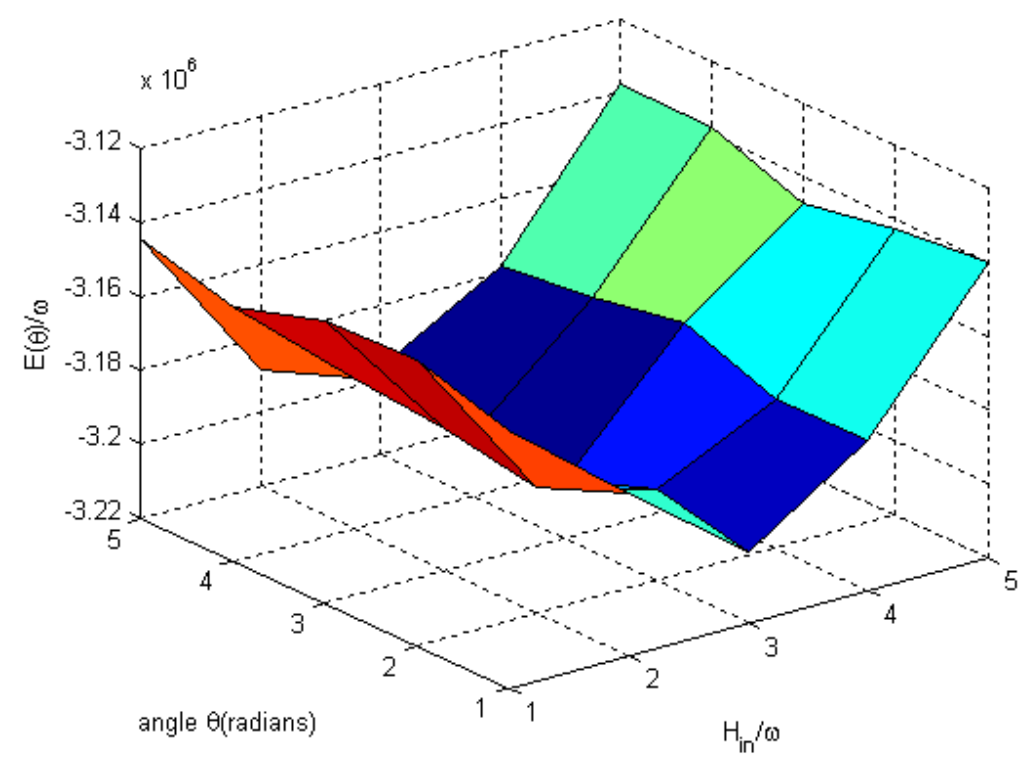

Figure 3 : 3-D graph of $\frac{E(\theta)}{\omega}$ versus $\frac{H_{\text {in }}}{\omega}$ and angle for $\operatorname{sc}(001)$ film with $\mathrm{N}=1000$ when the in plane applied magnetic field is zero.

$$
\frac{\boldsymbol{E}(\boldsymbol{\theta})}{\boldsymbol{\omega}}=-3168 \times 10^{3}+3141 \cos 2 \theta-4000 \cos ^{2} \theta-189 \cos ^{4} \theta-1000\left(\frac{\boldsymbol{H}_{\text {in }}}{\boldsymbol{\omega}} \sin \theta+5 \sin 2 \theta\right)
$$

The 3-D graph of $\frac{\boldsymbol{E}(\boldsymbol{\theta})}{\boldsymbol{\omega}}$ versus $\frac{\boldsymbol{H}_{\text {in }}}{\boldsymbol{\omega}}$ and angle is given in figure 3. According to this graph, the film with 1000 layers can be easily oriented in some certain directions by applying an external magnetic field of $\frac{\boldsymbol{H}_{\boldsymbol{i n}}}{\boldsymbol{\omega}}=4$.8. Although the energy varies in the same range as previous graph, the variation of energy is different from the previous graph. The graph between energy and angle at $\frac{\boldsymbol{H}_{\boldsymbol{i n}}}{\boldsymbol{\omega}}=4.8$ is given in figure 4 . The energy becomes minimum and maximum at $57.6^{\circ}$ and $147.7^{\circ}$, respectively. The film can be easily oriented in the direction of $57.6^{\circ}$ by applying this field of $\frac{\boldsymbol{H}_{\boldsymbol{i n}}}{\boldsymbol{\omega}}=4.8$ in the plane of the film. 


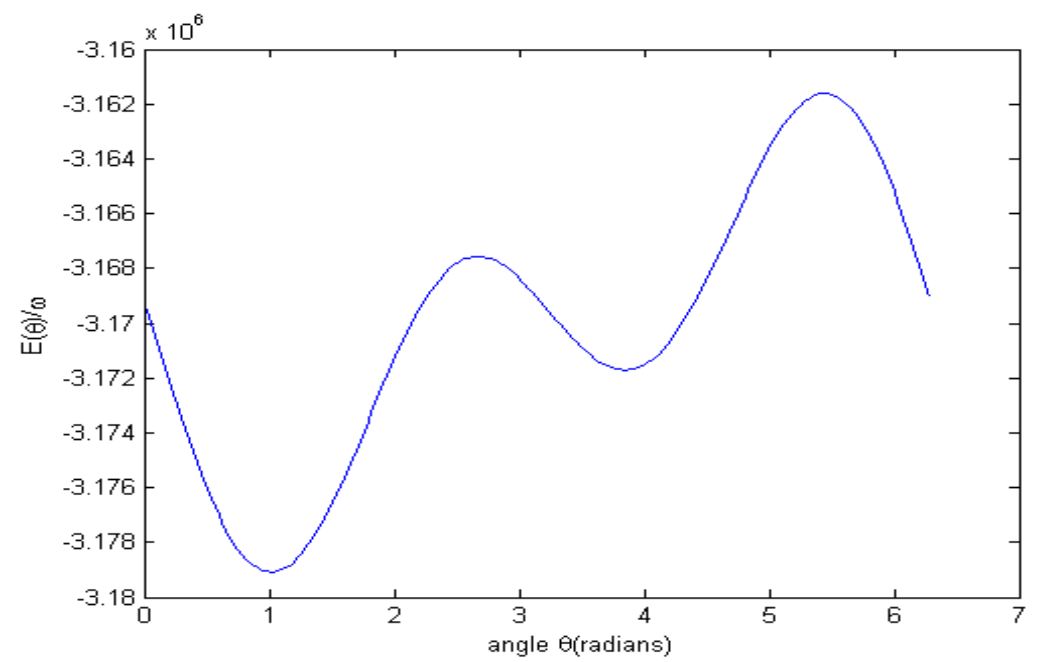

Figure 4 : Graph between energy and angle at $\frac{H_{\text {in }}}{\omega}=4.8$

When, $\frac{\boldsymbol{N}_{\boldsymbol{d}}}{\boldsymbol{\mu}_{\mathbf{0}} \boldsymbol{\omega}}=\mathbf{1 0}, \frac{\sum_{m=1}^{N} D_{m}{ }^{(2)}}{\omega}=4000, \frac{\sum_{m=1}^{N} D_{m}{ }^{(4)}}{\omega}=189, \frac{\boldsymbol{H}_{\text {in }}}{\boldsymbol{\omega}}=\mathbf{5}$ and $\frac{\boldsymbol{H}_{\text {out }}}{\boldsymbol{\omega}}=\mathbf{5}$ $\frac{\boldsymbol{E}(\boldsymbol{\theta})}{\boldsymbol{\omega}}=-3168 \times 10^{3}+3141 \cos 2 \boldsymbol{\theta}-4000 \cos ^{2} \theta-189 \cos ^{4} \theta-1000\left(5 \sin \theta+5 \cos +\frac{\boldsymbol{K}_{s}}{\boldsymbol{\omega}} \sin 2 \theta\right)$

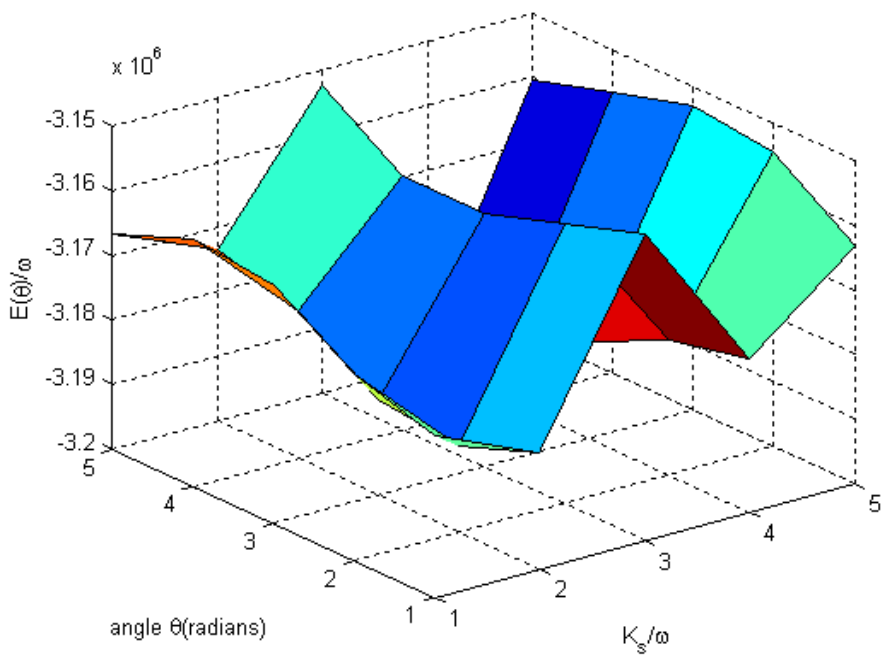

Figure 5 : $\quad 3-\mathrm{D}$ graph of $\frac{E(\theta)}{\omega}$ versus $\frac{K_{s}}{\omega}$ and angle for sc(001) with 1000 layers. 
The 3-D graph of $\frac{\boldsymbol{E}(\boldsymbol{\theta})}{\boldsymbol{\omega}}$ versus $\frac{\boldsymbol{K}_{s}}{\boldsymbol{\omega}}$ and angle is given in figure 5. The graph indicates some minimum energy values at some certain angles and some certain stress values. For example, the film can be easily oriented in direction of $172^{\circ}$ by applying the stress $\frac{\boldsymbol{K}_{s}}{\boldsymbol{\omega}}=3.6$.

Using $\mathrm{Z}_{0}=0, \mathrm{Z}_{1}=4, \Phi_{0}=5.8675, \Phi_{1}=2.7126, \mathrm{~b}=\pi$ and $a=6 \sqrt{3} \pi^{2}$ for $\mathrm{bcc}(001)$ lattice in equation number 2 (Usadel \& Hucht, 2002),

$$
\begin{aligned}
& \mathrm{E}(\theta)=-4240 \times 10^{3} \omega+1465 \omega(1+3 \cos 2 \theta) \\
& -\cos ^{2} \theta \sum_{m=1}^{N} D_{m}{ }^{(2)}-\cos ^{4} \theta \sum_{m=1}^{N} D_{m}{ }^{(4)}-1000\left(H_{\text {in }} \sin \theta+H_{\text {out }} \cos \theta-\frac{N_{d}}{\mu_{0}}+K_{s} \sin 2 \theta\right) \\
& \text { When, } \frac{\boldsymbol{N}_{\boldsymbol{d}}}{\boldsymbol{\mu}_{\mathbf{0}} \boldsymbol{\omega}}=\mathbf{1 0}, \frac{\sum_{m=1}^{N} D_{m}{ }^{(2)}}{\omega}=4000, \frac{\sum_{m=1}^{N} D_{m}{ }^{(4)}}{\omega}=189, \mathrm{H}_{\mathrm{in}}=0 \text { and } \frac{\boldsymbol{K}_{s}}{\omega}=\mathbf{5} \\
& \frac{\boldsymbol{E}(\boldsymbol{\theta})}{\boldsymbol{\omega}}=-4229 \times 10^{3}+4395 \cos 2 \theta-4000 \cos ^{2} \theta-189 \cos ^{4} \theta-1000\left(\frac{\boldsymbol{H}_{\text {out }}}{\omega}\right. \\
& \cos \theta+5 \sin 2 \theta)
\end{aligned}
$$

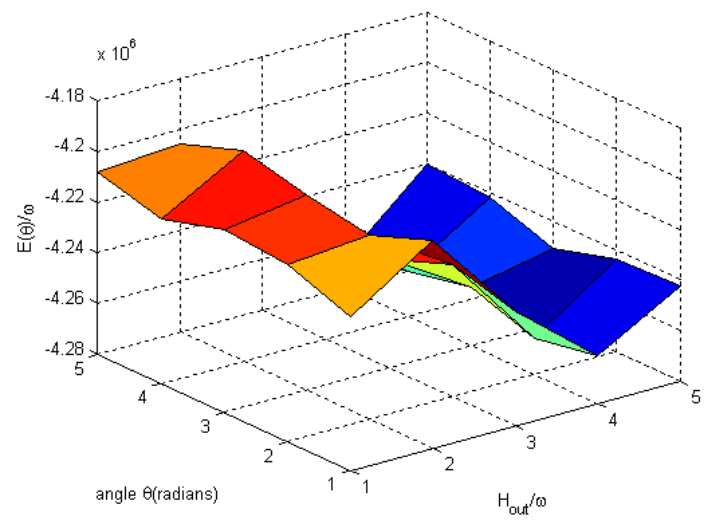

Figure 6 : 3-D graph of $\frac{E(\theta)}{\omega}$ versus $\frac{H_{\text {out }}}{\omega}$ and angle for bcc(001) with 1000 layers. 
The 3-D graph of $\frac{\boldsymbol{E}(\boldsymbol{\theta})}{\boldsymbol{\omega}}$ versus $\frac{\boldsymbol{H}_{\text {out }}}{\boldsymbol{\omega}}$ and angle is given in figure 6. Although the range of energy is different from that of $\operatorname{sc}(001)$ lattice, the variation of energy is almost the same as that of sc(001) film.

\section{CONCLUSIONS}

The energy required to orient the film in some certain directions becomes minimum at $\frac{\boldsymbol{H}_{\text {out }}}{\boldsymbol{\omega}}=4.8$ or $\frac{\boldsymbol{H}_{\text {in }}}{\boldsymbol{\omega}}=4.8$. The film with 1000 layers can be easily oriented along directions of $41.4^{\circ}$ or $57.6^{\circ}$ by applying this magnetic field perpendicular or in plane directions, respectively. The film can be easily oriented along direction of $172^{\circ}$ by applying the external stress $\frac{\boldsymbol{K}_{s}}{\boldsymbol{\omega}}=3.6$ in perpendicular direction. The values of angle of orientation, in plane magnetic field, out of plane magnetic field and stress corresponding to energy minimum and maximums are almost same for both sc(001) and bcc(001) lattice types. But the values of energies at maxima and minima are slightly different for these two types of lattices. Although this simulation was performed for some selected values of $\frac{J}{\omega}, \frac{N_{d}}{\mu_{0} \omega}, \frac{\sum_{m=1}^{N} D_{m}{ }^{(2)}}{\omega}, \frac{\sum_{m=1}^{N} D_{m}{ }^{(4)}}{\omega}, \frac{\boldsymbol{H}_{\text {out }}}{\boldsymbol{\omega}}$, $\frac{\boldsymbol{H}_{\text {in }}}{\boldsymbol{\omega}}$ and $\frac{K_{s}}{\omega}$ only, the same simulation can be carried out for any vales of above parameters.

\section{REFERENCES}

Bentaleb, M., N. El Aouad \& M. Saber. 2002. Magnetic properties of the spin $-1 / 2$ Ising Ferromagnetic thin films with alternating superlattice configuration. Chinese Journal of Physics 40(3): 307-314.

Jensen, P.J. \& H. Dreysse. 2002. In-plane magnetic reorientation in coupled ferro and antiferromagnetic thin films. Physical Review B 66: 220407-1-4.

Samarasekara, P. 2006. A solution of the Heisenberg Hamiltonian for oriented thick ferromagnetic films. Chinese Journal of Physics 44(5): 377-386.

Samarasekara, P. \& F.J. Cadieu. 2001. Polycrystalline Ni ferrite films deposited by RF sputtering techniques. Japanese Journal of Applied Physics 40: 3176-3179. 


\section{P Samarasekara}

Samarasekara, P. \& F.J. Cadieu. 2001. Magnetic and Structural Properties of RF Sputtered Polycrystalline Lithium Mixed Ferrimagnetic Films. Chinese Journal of Physics 39(6): 635-640.

Shan-Ho, Tsai, D.P. Landau \& C. Thomas Schulthess. 2003. Effect of interfacial coupling on the magnetic ordering in ferro-antiferromagnetic bilayers. Journal of Applied Physics 93(10): 8612-8614.

Usadel, K.D. \& A. Hucht. 2002. Anisotropy of ultrathin ferromagnetic films and the spin reorientation transition. Physical Review B 66: 024419-1-6.

Ze-Nong, Ding, D.L. Lin \& Libin Lin. 1993. Surface magnetism in a thin film of Heisenberg Ferrrimagnets. Chinese Journal of Physics 31(3): 431-440.

Zhao, D., Feng Liu, D.L. Huber \& M.G. Lagally. 2002. Step-induced magnetichysteresis anisotropy in ferromagnetic thin films. Journal of Applied Physics 91 (5): 3150-3153. 